\title{
Are Orthogonal Separable Coordinates Really Classified?^
}

Konrad SCHÖBEL

Mathematisches Institut, Fakultät für Mathematik und Informatik, Friedrich-Schiller-Universität Jena, 07737 Jena, Germany

E-mail: konrad.schoebel@uni-jena.de

Received October 30, 2015, in final form March 15, 2016; Published online April 26, 2016

http://dx.doi.org/10.3842/SIGMA.2016.041

\begin{abstract}
We prove that the set of orthogonal separable coordinates on an arbitrary (pseudo-)Riemannian manifold carries a natural structure of a projective variety, equipped with an action of the isometry group. This leads us to propose a new, algebraic geometric approach to the classification of orthogonal separable coordinates by studying the structure of this variety. We give an example where this approach reveals unexpected structure in the well known classification and pose a number of problems arising naturally in this context.
\end{abstract}

Key words: separation of variables; Stäckel systems; Deligne-Mumford moduli spaces; Stasheff polytopes; operads

2010 Mathematics Subject Classification: 14H70; 53A60; 58D27

\section{Introduction}

"Of course, they are!" any reader familiar with the topic will immediately reply to the question in the title, "The problem has been solved exhaustively almost 30 years ago". Indeed, based on the pioneering works of Paul Stäckel [31] and Luther P. Eisenhart [8], Ernest G. Kalnins and Willard Miller gave a comprehensive solution of the classical problem to classify all orthogonal separable coordinate systems on a constant curvature manifold [13, 15]. These are coordinates in which fundamental equations like the Hamilton-Jacobi equation or the Schrödinger equation can be solved by a separation of variables. With our somewhat provocative title we do by no means at all want to cast any doubt on this milestone in the theory of separation of variables, but rather question what is meant by the word solved. Our reader will again reply that Kalnins and Miller's result gives a complete list of (families of equivalence classes of) orthogonal separable coordinates. The issue we would like to address here is that this list, mathematically speaking, is nothing but a mere set, whereas we will show that the combination of some very elementary observations leads to the conclusion that this set actually has a rich geometric structure. Namely, we prove:

Main Theorem. The set of orthogonal separable coordinates on an n-dimensional (pseudo-)Riemannian manifold $M$ carries a natural structure of a projective variety, isomorphic to a subvariety $\mathcal{S}(M) \subseteq G_{n}(\mathcal{K}(M))$ in the Grassmannian of $n$-planes in the space $\mathcal{K}(M)$ of second order Killing tensors on $M$. Moreover, it comes equipped with a natural action of the isometry group.

We call $\mathcal{S}(M)$ the variety of orthogonal separable coordinates on $M$.

Our theorem shows that the classification problem for orthogonal separable coordinates is, essentially, an algebraic geometric problem and should therefore be treated by algebraic geometric rather than differential geometric methods. To the best of our knowledge, this point

\footnotetext{
${ }^{\star}$ This paper is a contribution to the Special Issue on Analytical Mechanics and Differential Geometry in honour of Sergio Benenti. The full collection is available at http://www.emis.de/journals/SIGMA/Benenti.html
} 
has never been made in the vast amount of literature on separation of variables. Separable coordinates have always been studied via partial differential equations, while our result implies that it is governed by algebraic equations alone. This observation could have been made over 60 years ago, since it is mainly a consequence of the Nijenhuis integrability conditions, published in 1951 [24].

So, from a categorical point of view, we can say that the classification problem for orthogonal separable coordinates is solved, but in the category of sets and not in its natural category, which is the category of projective varieties equipped with group actions. This motivates us to propose an algebraic geometric approach to the classification of orthogonal separable coordinates, by investigating the variety $\mathcal{S}(M)$ as in [29] for constant curvature manifolds.

Main Problem. For a given (pseudo-)Riemannian manifold $M$, describe the structure of the variety $\mathcal{S}(M)$ of orthogonal separable coordinate systems and of its quotient $\mathcal{S}(M) / G$ under the isometry group $G$.

What does it mean to describe the structure of this variety and why should that give any relevant information for the classification of separation coordinates? We will illustrate this using the example of the $n$-dimensional sphere $\mathbb{S}^{n}$, for which the variety of orthogonal separable coordinates in normal form has recently been identified to the Deligne-Mumford moduli space $\overline{\mathscr{M}}_{0, n+2}(\mathbb{R})$ of stable algebraic curves of genus zero with $n+2$ marked points [30]. Exploiting the properties of these spaces reveals a rich and unexpected geometric, combinatorial and algebraic structure behind the long known classification.

While some may have considered separation of variables as "old-fashioned" or even "outdated", this example builds a bridge from the over 120 years old theory of separation of variables to modern algebraic geometry and popular topics like operad theory. Spheres are but the simplest example of constant curvature manifolds in this context and even more interesting results are expected for other manifolds and other notions of variable separation. That is to say, we are far from a complete understanding of variable separation in all its facets.

To keep this notice concise, we will refrain from giving a more elaborate introduction to separation of variables, which can be found elsewhere in the abundant literature on this subject. We concentrate instead on those few definitions and results necessary to set up our algebraic geometric description.

\section{Some simple observations}

\subsection{Killing tensors}

Our first two observations concern second order Killing tensors, the fundamental object in the characterisation of separable coordinates. These are symmetric tensor fields $K_{\alpha \beta}$ on a (pseudo-)Riemannian manifold which satisfy the Killing equation

$$
\nabla_{a} K_{b c}+\nabla_{b} K_{c a}+\nabla_{c} K_{a b}=0 .
$$

Here $\nabla$ denotes the covariant derivative with respect to the Levi-Civita connection of the metric. Note that the metric itself is trivially a Killing tensor, since it is covariantly constant.

Observation 1. The Killing equation (2.1) is linear. Therefore Killing tensors form a vector space.

Observation 2. The Killing equation (2.1) is overdetermined of finite type. Hence the space of Killing tensors is finite-dimensional.

Definition 1. We denote the finite-dimensional space of second order Killing tensors on a (pseudo-)Riemannian manifold $M$ by $\mathcal{K}(M)$. 


\subsection{Stäckel systems}

Here and in what follows we will silently identify symmetric forms with endomorphisms using the metric. In particular, we can interpret a Killing tensor as a field of symmetric endomorphisms. We say that two Killing tensors commute if the corresponding endomorphisms commute algebraically at every point. Note that the metric corresponds to the identity endomorphism and hence commutes with any other Killing tensor.

A field of symmetric endomorphisms is said to have surface normal eigenvectors, if it has simple eigenvalues and the orthogonal complement of each eigenvector field forms an integrable distribution ${ }^{1}$. By definition such a field defines a family of $n$ orthogonal hypersurface foliations. Moreover, any symmetric endomorphism field with surface normal eigenvectors and commuting with the first defines the same foliations.

Definition 2. A Stäckel system on an $n$-dimensional (pseudo-)Riemannian manifold is an $n$ dimensional vector space spanned by the metric and $n-1$ mutually commuting Killing tensors with surface normal eigenvectors.

The leaves of a family of $n$ complementary hypersurface foliations are the coordinate hypersurfaces of a coordinate system which is unique up to permutations and reparametrisations of the individual coordinates. By abuse of language, we will call an equivalence class of coordinate systems under these transformations simply coordinates. Moreover, if the Hamilton-Jacobi equation

$$
\frac{1}{2} g^{i j} \frac{\partial W}{\partial x^{i}} \frac{\partial W}{\partial x^{j}}=E
$$

admits a solution of the form

$$
W\left(x^{1}, \ldots, x^{n} ; \underline{c}\right)=W_{1}\left(x^{1} ; \underline{c}\right)+\cdots+W_{n}\left(x^{n} ; \underline{c}\right), \quad \operatorname{det}\left(\frac{\partial^{2} W}{\partial x^{i} \partial c_{j}}\right) \neq 0
$$

in the coordinates $x^{1}, \ldots, x^{n}$, we call them separable coordinates. We can now state the classical result, which is the basis for their classification.

Theorem 1 ([8,31]). Locally, there is a bijective correspondence between Stäckel systems and orthogonal separable coordinates for the Hamilton-Jacobi equation.

Although the above correspondence is only local, we can use it to classify orthogonal separable coordinates which are global in the sense that they are induced by a global Stäckel system. In particular, it gives a global classification if every local Killing tensor extends to a global one, as is the case with simply connected constant curvature manifolds.

We should remark that an (additive) separation of the Hamilton-Jacobi equation is a necessary condition for a (multiplicative) separation of the Schrödinger and other prominent equations and that sufficient conditions can be given $[8,26]$. This is why the classical result is formulated for the Hamilton-Jacobi equation. We conclude with an algebraic geometric view on Stäckel systems.

Observation 3. By definition, a Stäckel system defines a point in the Grassmannian $G_{n}(\mathcal{K}(M))$ of n-planes in the space of Killing tensors.

\footnotetext{
${ }^{1}$ Some care has to be taken on a pseudo-Riemannian manifold, where the eigenvalues and eigenvectors may be complex. In this case either the separable coordinates are not everywhere defined or one weakens the notion of variables separation to allow for complex variables.
} 


\subsection{Nijenhuis integrability}

The above geometric definition of surface normal eigenvectors is not suitable for our purposes. We will instead use an analytic characterisation due to Nijenhuis [24], who showed that a diagonalisable endomorphism field $K$ has surface normal eigenvectors if and only if it satisfies the following Nijenhuis integrability conditions:

$$
\begin{aligned}
& K_{d[a} \nabla_{b} K_{c]}{ }^{d}=0, \\
& K_{d}{ }^{e} K_{e[a} \nabla_{b} K_{c]}{ }^{d}+K_{d[a} K_{b}^{e} \nabla^{d} K_{c] e}=0, \\
& K_{d}{ }^{e} K_{e}{ }^{f} K_{f[a} \nabla_{b} K_{c]}{ }^{d}+K_{e}{ }^{f} K_{d[a} K_{b}^{e} \nabla^{d} K_{c] f}=0 .
\end{aligned}
$$

Definition 3. We call a Killing tensor satisfying the Nijenhuis integrability conditions (2.2) Nijenhuis integrable and denote the set of Nijenhuis integrable Killing tensors on a (pseudo-)Riemannian manifold $M$ by $\mathcal{I}(M)$.

The next observation is due to Sergio Benenti [1].

Observation 4. Every Killing tensor with surface normal eigenvectors is contained in a unique Stäckel system.

Note that this observation would reduce the classification of separable coordinates to solving the Nijenhuis conditions for a (single) Killing tensor, since the determination of the corresponding Stäckel system is only a linear problem. Nevertheless, a direct solution of this system of nonlinear partial differential equations has been considered intractable [11]. In contrast, our next two observations show that these equations have a remarkable property.

Observation 5. The covariant derivative $K \mapsto \nabla K$ is a linear operation. Hence any polynomial expression in $K$ and $\nabla K$ is actually polynomial in $K$ alone.

Observation 6. The Nijenhuis equations are homogeneous algebraic equations in (the components of) $K$ and its covariant derivative $\nabla K$. This implies that the Nijenhuis integrability conditions define polynomial equations on the space $\mathcal{K}(M)$ of Killing tensors.

Let us reformulate this in algebraic geometric terms.

Observation $\mathbf{6}^{\prime}$. The set $\mathcal{I}(M)$ of Nijenhuis integrable Killing tensors forms a projective variety in the space $\mathcal{K}(M)$ of Killing tensors.

A priori, the Nijenhuis conditions yield infinitely many algebraic equations on $\mathcal{K}(M)$, as they have to be satisfied at every point of $M$. By Hilbert's basis theorem these can be reduced to a finite set. For an explicit example see [27]. Note that we will not, as usually done, exclude Nijenhuis integrable Killing tensors with multiple eigenvalues since this would destroy the property of $\mathcal{I}(M)$ to be a variety.

By definition, a Stäckel system is a projective $(n-1)$-plane contained in the variety $\mathcal{I}(M)$. Observation 4 then tells us that $\mathcal{I}(M)$ has a very particular structure: It is ruled by $(n-1)$ planes. For an explicit example see [28].

\subsection{Isometry group action}

We seek to classify separable coordinates up to isometries. We will therefore conclude this section with three observations concerning the isometry group action.

Observation 7. The Killing equation (2.1) is invariant under isometries, so that the isometry group acts linearly on the space of Killing tensors. 
Observation 8. The Nijenhuis integrability conditions (2.2) and therefore the set $\mathcal{I}(M)$ of Nijenhuis integrable Killing tensors are invariant under isometries.

Observation 9. The property that two Killing tensors commute is invariant under isometries.

Combining the preceding two observations, we arrive at:

Observation 10. The isometry group maps Stäckel systems to Stäckel systems.

\section{The foundation}

We now put together all observations made so far in order to prove our Main Theorem. We will employ three standard results from algebraic geometry:

Fact 1 ([9]). The set of pairs of nested planes of fixed dimensions $k_{1} \leq k_{2}$ in a vector space $V$,

$$
\Psi_{k_{1}, k_{2}}(V):=\left\{\left(U_{1}, U_{2}\right): U_{1} \subseteq U_{2}\right\} \subset G_{k_{1}}(V) \times G_{k_{2}}(V),
$$

is a subvariety of the product of the corresponding Grassmannians. It comes with two projections onto the factors,

$$
G_{k_{1}}(V) \stackrel{\pi_{1}}{\longleftarrow} \Psi_{k_{1}, k_{2}}(V) \stackrel{\pi_{2}}{\longrightarrow} G_{k_{2}}(V)
$$

Fact 2 ([9]). The set of $n$-planes contained in a given projective variety $X \subseteq \mathbb{P}^{d}$ forms a subvariety in the Grassmannian of n-planes in $\mathbb{P}^{d}$, called the Fano variety $F_{n}(X) \subseteq G_{n}\left(\mathbb{P}^{d}\right)$ of $X$.

Fact 3. The maximal commutative subalgebras consisting of symmetric endomorphisms of an ndimensional vector space $V$ form a subvariety $\mathcal{C}(V) \subseteq G_{n}\left(S^{2} V\right)$ in the Grassmannian of n-planes in $S^{2} V$.

Proof. For definite metrics the statement simply follows from the closed orbit lemma [2]. Indeed, the space of diagonal endomorphisms defines a point in $G_{n}\left(S^{2} V\right)$ and $\mathcal{C}(V)$ is the orbit of this point under the action of the algebraic group $\mathrm{SO}(V)$. For indefinite metrics we have finitely many normal forms for maximal commuting subalgebras, each of them defining a point in $G_{n}\left(S^{2} V\right)$. By the closed orbit lemma, the orbit of each of these points defines a subvariety. Since $\mathcal{C}(V)$ is the union of these orbits, it is a union of finitely many subvarieties and hence itself a subvariety.

Our Main Theorem will be a simple consequence of the following lemma.

Lemma 1. Let $X \subseteq G_{n}\left(V_{1}\right)$ and $Y \subseteq G_{n}\left(V_{1} \oplus V_{2}\right)$ be subvarieties. Then the set $Z=\{y \in Y$ : $\left.\exists x \in X: p_{1}(y) \subseteq x\right\}$, where $p_{1}$ is the projection onto the first factor, is a projective variety.

Proof. First note that the condition $p_{1}(y) \subseteq x$ is equivalent to $y \subseteq p^{-1}(x)=x \oplus V_{2}$. Mapping $x \mapsto x \oplus V_{2}$ gives an embedding $\iota: G_{n}\left(V_{1}\right) \hookrightarrow G_{m}\left(V_{1} \oplus V_{2}\right)$, where $m=n+\operatorname{dim} V_{2}$. Indeed, taking $k_{1}=\operatorname{dim} V_{2} \leq k_{2}=m$ and $V=V_{1} \oplus V_{2}$ in Fact 1 , the image is the variety $\pi_{2}\left(\pi_{1}^{-1}\left(\left\{0 \oplus V_{2}\right\}\right)\right)$. Applying Fact 1 once again, this time for $k_{1}=n \leq k_{2}=m$, we obtain the variety $Z=$ $\pi_{1}\left(\pi_{2}^{-1}(\iota(X))\right) \cap Y$.

Proof of the Main Theorem. Fact 2 implies that all $n$-planes contained in the variety $\mathcal{I}(M)$ $\subseteq \mathcal{K}(M)$ form a subvariety $F_{n}(\mathcal{I}(M)) \subseteq G_{n}(\mathcal{K}(M))$. These $n$-planes comprise Stäckel systems, but not all of them are Stäckel systems. We have to prove that the Stäckel systems among them form a subvariety. This results from Fact 3 as follows. We have already mentioned, that the 
Killing equation (2.1) is overdetermined of finite type. To be more precise, all derivatives of a Killing tensor $K_{a b}$ can be expressed as linear combinations of

$$
\begin{aligned}
& K_{a b} \in \Gamma\left(\varpi T^{*} M\right), \\
& \nabla_{a} K_{b c} \in \Gamma\left(\boxplus T^{*} M\right), \\
& Y\left(\nabla_{a} \nabla_{c} K_{b d}\right) \in \Gamma\left(\boxplus T^{*} M\right),
\end{aligned}
$$

the coefficients being polynomial in the Riemannian curvature tensor [36]. Here

- $\square T^{*} M=S^{2} T^{*} M$ is the bundle of symmetric forms $K_{a b}$ on $M$;

- $\square T^{*} M$ denotes the bundle of tensors $T_{a b c}$ on $M$ with the symmetries

$$
T_{a c b}=T_{a b c}, \quad T_{a b c}+T_{b c a}+T_{c a b}=0 ;
$$

- $\boxplus T^{*} M$ denotes the bundle of tensors $R_{a b c d}$ with curvature tensor symmetry and $Y$ stands for the corresponding Young projector (given by symmetrising in $a, c$ and $b, d$, followed by an antisymmetrisation in $a, b$ and $c, d)$.

This gives a linear evaluation map

$$
\mathrm{ev}_{p}: \mathcal{K}(M) \longrightarrow \mathcal{T}_{p}
$$

for each point $p \in M$, where

$$
\mathcal{T}_{p}:=\square T_{p}^{*} M \oplus \boxplus T_{p}^{*} M \oplus \boxplus T_{p}^{*} M
$$

is the fibre over $p$ of the tractor bundle $\mathcal{T} M$ corresponding to the prolongation of the Killing equation (2.1). This map is injective and gives an embedding

$$
\iota: G_{n}(\mathcal{K}(M)) \hookrightarrow G_{n}\left(\mathcal{T}_{p}\right) .
$$

On the other hand, by Fact 3 we have an inclusion $\mathcal{C}\left(T_{p}^{*} M\right) \subseteq G_{n}\left(\varpi T_{p}^{*} M\right)$. We can now apply the above lemma to the varieties

$$
X=\mathcal{C}\left(T_{p}^{*} M\right) \subseteq G_{n}\left(\square T_{p}^{*} M\right), \quad Y=\iota\left(F_{n}(\mathcal{I}(M))\right) \subseteq G_{n}\left(\mathcal{T}_{p}\right)
$$

to obtain a variety $Z \subseteq G_{n}\left(\mathcal{T}_{p}\right)$. Its pull back $\iota^{-1}(Z) \subseteq G_{n}(\mathcal{K}(M))$ under the embedding (3.1) consists of all $n$-planes of Nijenhuis integrable Killing tensors whose members commute at the fixed point $p$. The intersection of these varieties for all $p \in M$ consists of $n$-planes of Nijenhuis integrable Killing tensors whose members commute at every point $p \in M$. By definition, these are the Stäckel systems on $M$. This proves the first part of the Main Theorem.

By Observation 10 the variety $\mathcal{S}(M)$ is invariant under the induced action of the isometry group on $G_{n}(\mathcal{K}(M))$. This proves the second part.

\section{The proof of concept}

Let $M=\mathbb{S}^{n} \subset \mathbb{R}^{n+1}$ denote the standard round sphere of dimension $n$. It has the orthogonal group $\mathrm{O}(n+1)$ as isometry group with Lie algebra

$$
\mathfrak{s o}(n+1)=\left\langle e_{i} \wedge e_{j}: 0 \leq i<j \leq n\right\rangle .
$$

Since the space of Killing tensors on constant sectional curvature spaces is known to be generated by symmetric products of Killing vectors, we can embed the space of Killing tensors into the space of symmetric forms on the Lie algebra of the isometry group,

$$
\mathcal{K}\left(\mathbb{S}^{n}\right) \subseteq S^{2} \mathfrak{s o}(n+1) .
$$




\subsection{Normal forms}

While $\mathcal{S}(M)$ is a variety, this property will in general be lost for the quotient $\mathcal{S}(M) / G$. As a remedy, one could consider the geometric invariant theory quotient $\mathcal{S}(M) / / G$ instead or try to find a slice for the $G$-action, i.e., a subvariety $\mathcal{S}_{0}(M) \subset \mathcal{S}(M)$ with finite stabiliser which intersects every group orbit transversally. In the latter case we would still deal with a variety which parametrises isometry classes of separable coordinates, but trade this off against having a unique parametrisation. This is acceptable, since the ambiguity is only finite. In the case of spheres, such a slice is provided by the following result.

Theorem 2 ([3]). Up to an isometry every Stäckel system is contained in

$$
\mathcal{K}_{0}\left(S^{n}\right):=\left\langle\left(e_{i} \wedge e_{j}\right)^{2}: 0 \leq i<j \leq n\right\rangle .
$$

Note that $\mathcal{K}_{0}\left(S^{n}\right)$ is the space of diagonal symmetric forms on the Lie algebra $\mathfrak{s o}(n+1)$ with respect to the basis $\left\{e_{i} \wedge e_{j}\right\}$.

Definition 4. We call $\mathcal{K}_{0}\left(S^{n}\right)$ the subspace of Killing tensors on $\mathbb{S}^{n}$ in normal form and define

$$
\mathcal{S}_{0}\left(\mathbb{S}^{n}\right):=\mathcal{S}\left(\mathbb{S}^{n}\right) \cap G_{n}\left(\mathcal{K}_{0}\left(\mathbb{S}^{n}\right)\right)
$$

to be the variety of separable coordinates in normal form.

It is not difficult to figure out that the stabiliser of $\mathcal{K}_{0}\left(S^{n}\right)$ in $\mathrm{O}(n+1)$ is the hyperoctahedral group $S_{n+1} \ltimes \mathbb{Z}_{2}{ }^{n+1}$, which acts by permutations and sign changes of the fixed basis $\left\{e_{i}\right\}$, and that the stabiliser action on $\mathcal{K}_{0}\left(S^{n}\right)$ descends to the permutation group $S_{n+1}$. We therefore have

$$
\frac{\mathcal{S}\left(\mathbb{S}^{n}\right)}{\mathrm{O}(n+1)} \cong \frac{\mathcal{S}_{0}\left(\mathbb{S}^{n}\right)}{S_{n+1}}
$$

That is, we replace the quotient of a variety by a continuous group with a quotient of a linear section of this variety by a finite group.

\subsection{The isomorphism}

Recall our Main Problem, to describe the structure of the variety $\mathcal{S}(M)$ and the isometry group action on it. In the preceding section we reduced this problem for $M=\mathbb{S}^{n}$ to describing the variety $\mathcal{S}_{0}\left(\mathbb{S}^{n}\right)$ and the $S_{n+1}$-action on it. The following theorem solves our Main Problem at once, by identifying $\mathcal{S}_{0}\left(\mathbb{S}^{n}\right)$ to a complicated, but well understood moduli space.

"In great mathematics there is a very high degree of unexpectedness, combined with inevitability and economy."

GODFREY HAROLD HARDY

Theorem 3 ([30]). The variety of Stäckel systems on $\mathbb{S}^{n}$ in normal form is a smooth (!) projective variety, isomorphic to the real part of the Deligne-Mumford moduli space of stable curves of genus 0 with $n+2$ marked points:

$$
\mathcal{S}_{0}\left(\mathbb{S}^{n}\right) \cong \overline{\mathscr{M}}_{0, n+2}(\mathbb{R}) .
$$

This isomorphism is $S_{n+1}$-equivariant. 
To give this theorem a meaning, one would have to say some words about the moduli spaces $\overline{\mathscr{M}}_{0, n}$ and their real parts. We leave it at saying that $\overline{\mathscr{M}}_{0, n+1}(\mathbb{R})$ is a compactification of the configuration space of $n+1$ distinct points on $\mathbb{P}^{1}$ modulo automorphisms or, equivalently, of $n$ points on the real line modulo the affine group. Giving a formal definition would go far beyond the scope of this notice and we refer to the literature for more details on moduli spaces of curves $[5,10,18,19,22]$. To get an idea how these spaces look like, let us list the simplest examples.

- $\overline{\mathscr{M}}_{0,3}(\mathbb{R})$ is a point.

- $\overline{\mathscr{M}}_{0,4}(\mathbb{R})$ is a projective line.

- $\overline{\mathscr{M}}_{0,5}(\mathbb{R})$ is the blowup of a projective plane in four points.

- In general, $\overline{\mathscr{M}}_{0, n+2}(\mathbb{R})$ is an iterated blowup of $\mathbb{P}^{n-1}[4,6,17]$.

The isomorphism in Theorem 3 is rather surprising, as it relates two seemingly completely unrelated objects - separable coordinates for the Hamilton-Jacobi equation on one hand and stable algebraic curves with marked points on the other. We can exploit the isomorphism (4.2) by "pulling back" known properties from $\overline{\mathscr{M}}_{0, n+2}(\mathbb{R})$ to $\mathcal{S}_{0}\left(\mathbb{S}^{n}\right)$ and interpret them in terms of separable coordinates on spheres. In the rest of this section we will illustrate this by giving three examples of properties which reveal hitherto unknown structures in the long known classification of orthogonal separable coordinates.

\subsection{Stasheff polytopes}

A property which can readily be used to describe the quotient $\mathcal{S}\left(\mathbb{S}^{n}\right) / \mathrm{O}(n+1)$ is the fact that the moduli space $\overline{\mathscr{M}}_{0, n+2}(\mathbb{R})$ is tiled by $(n+1) ! / 2$ copies of the Stasheff polytope $K_{n+1}[6,17]$. Stasheff polytopes, or associahedra, were introduced by Stasheff as a combinatorial object in the homotopy theory of $H$-spaces [32, 33] ${ }^{2}$.

Instead of a formal definition, we list the simplest examples, which will be sufficient for our purpose of illustration:

- $K_{2}$ is a point;

- $K_{3}$ is a line segment;

- $K_{4}$ is a pentagon;

- in general, $K_{n+1}$ is an $(n-1)$-dimensional polytope.

Combining the homeomorphism (4.1) with the equivariant isomorphism (4.2), we obtain

$$
\frac{\mathcal{S}\left(\mathbb{S}^{n}\right)}{\mathrm{O}(n+1)} \cong \frac{\overline{\mathscr{M}}_{0, n+2}(\mathbb{R})}{S_{n+1}}
$$

The $S_{n+1}$-action on $\overline{\mathscr{M}}_{0, n+2}(\mathbb{R})$ is transitive on the $(n+1) ! / 2$ tiles and the stabiliser of a single tile is $\mathbb{Z}_{2}$, a reflection symmetry of the Stasheff polytope $K_{n+1}$. A fundamental domain for this action is therefore "one half" of the Stasheff polytope $K_{n+1}$ and the quotient (4.3) is obtained by identifying certain faces of $K_{n+1} / \mathbb{Z}_{2}$. In the next section we give the exact recipe which faces have to be identified.

\footnotetext{
${ }^{2}$ See also the historical note in Stasheff's contribution to [21].
} 

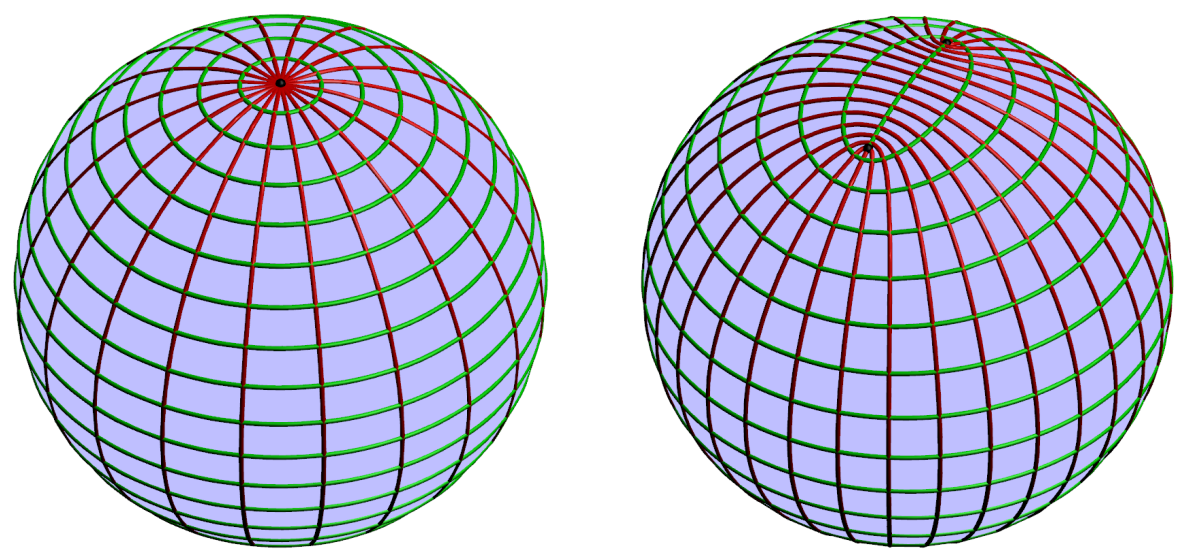

Figure 1. Spherical and elliptic coordinates on $\mathbb{S}^{2}$.

\subsection{Example}

On $\mathbb{S}^{2}$ there are two families of separable coordinates, as shown in Fig. 1: One is the familiar spherical coordinates, given by longitude and latitude. The other is elliptic coordinates, consisting of two families of confocal ellipses around two pairs of antipodal focal points. Modulo isometries, they form a one-parameter family, parametrised by the angular distance between the foci. Obviously, elliptic coordinates degenerate to spherical coordinates if this angle goes to 0 or $\pi$.

Fig. 2 shows how these two families are assembled to give the moduli space $\overline{\mathscr{M}}_{0,4}(\mathbb{R})$ tiled by three copies of $K_{3}$, which is a circle tiled by three intervals. The interiors of the three intervals correspond to elliptic coordinates whose focal points lie in one of the three coordinate planes, while the three boundary points separating them correspond to spherical coordinates with the poles on one of the three coordinate axes. When we cross the boundary between two intervals, the two pairs of foci first coalesce into one pair of poles, and then separate in orthogonal direction. Note that this trajectory is not smooth, which is why the smoothness of $\mathcal{S}_{0}\left(\mathbb{S}^{2}\right)$ comes rather unexpected.

Note also that there is a monodromy if we consider coordinates as ordered: If we start with spherical coordinates and track the longitudes while going once around the circle, we end up with latitudes and vice versa. This is illustrated in Fig. 2 by showing a two-fold cover (which is again a circle) and using a different colour for each coordinate.

The quotient $\mathcal{S}_{0}\left(\mathbb{S}^{2}\right) / S_{3}$ is an interval of which one endpoint corresponds to spherical coordinates, while the other one corresponds to elliptic coordinates where the four focal points are equidistantly spaced. In particular, these points are non-isometric and hence not identified in $K_{3} / \mathbb{Z}_{2}$.

Dimension $n=2$ is, however, trivial from our point of view, for in this case the Nijenhuis integrability conditions (2.2) are void, owing to the antisymmetrisation over three indices. For $n=3$ the tiling of $\overline{\mathscr{M}}_{0,5}(\mathbb{R})$ by twelve copies of $K_{4}$ can be seen as follows. Four general points in the projective plane determine six projective lines. These lines divide the plane into twelve triangles, each having two of the four points as vertices. A blowup in these four points therefore transforms the triangles into pentagons, which form the twelve tiles of $\overline{\mathscr{M}}_{0,5}(\mathbb{R})$.

\subsection{Rooted planar trees}

While points in $\mathcal{S}_{0}\left(\mathbb{S}^{n}\right)$ parametrise single isometry classes of separable coordinates, the (open) faces of a Stasheff polytope provide a more coarse classification into families. Stasheff polytopes have a number of remarkable combinatorial properties [6, 7], which can readily be used to label 


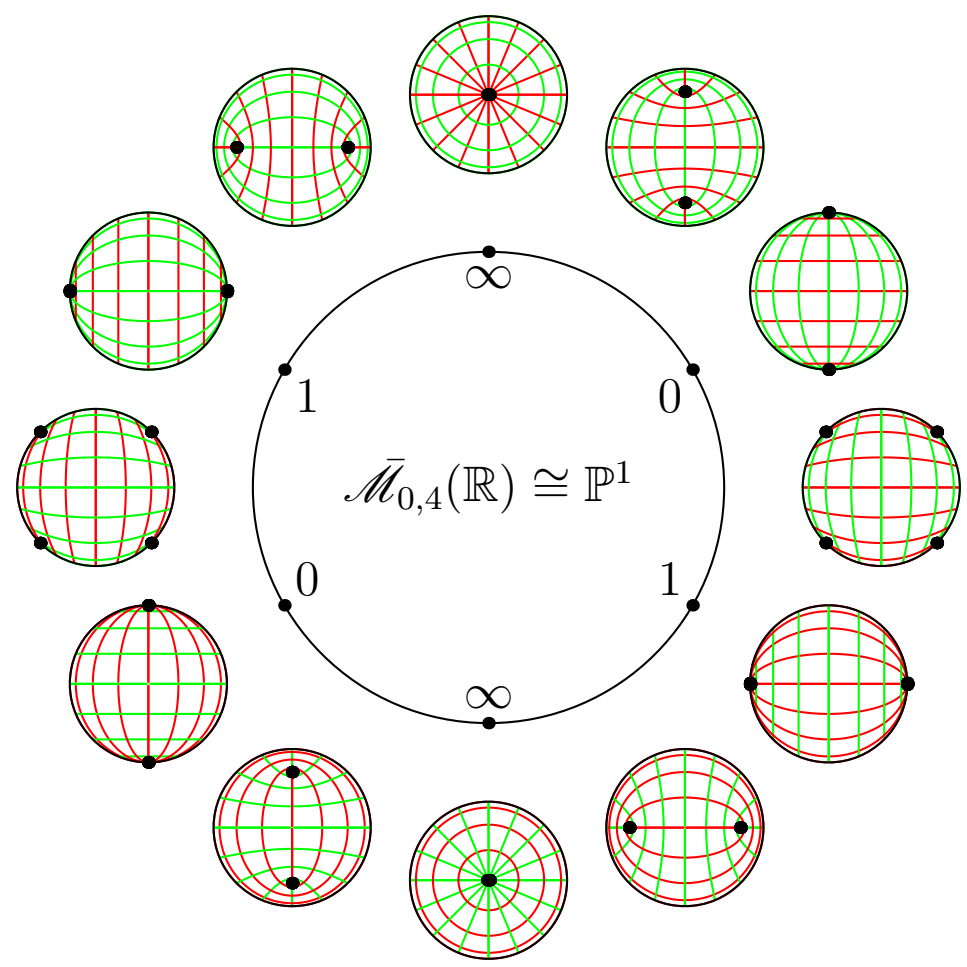

Figure 2. Parametrisation of separable coordinates in normal form on $\mathbb{S}^{2}$ by $\overline{\mathscr{M}}_{0,4}(\mathbb{R}) \cong \mathbb{P}^{1}$. To indicate the monodromy, a two-fold covering of the projective line is shown.

and count these families of separable coordinates.

- The faces of the Stasheff polytope $K_{n}$ can be labelled by rooted planar trees with $n$ leaves.

- The codimension $m$ faces correspond to trees with $m$ inner nodes other than the root. In particular, the number of vertices of $K_{n}$ is $C_{n-1}$, where $C_{n}=\frac{1}{n+1}\left(\begin{array}{c}2 n \\ n\end{array}\right)$ is the Catalan number.

We can therefore label the different families of orthogonal separable coordinates on spheres by rooted planar trees. For instance:

- Elliptic coordinates on $\mathbb{S}^{n}$, introduced by C. Neumann [23], form a family with $n-1$ continuous parameters, labelled by a corolla tree with $n+1$ leaves, as shown in Fig. 3 for $n+1=5$. They label the interior of the Stasheff polytope.

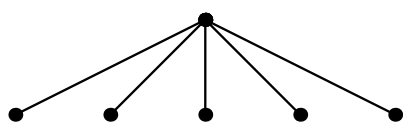

(a) corolla tree.

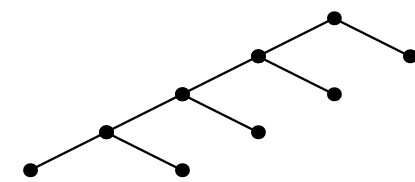

(b) left comb tree.

Figure 3.

- Polyspherical coordinates, introduced by Vilenkin [34], form a discrete family labelled by binary trees [35]. They correspond to the vertices of the Stasheff polytope.

- In particular, standard spherical coordinates correspond to a left comb tree, as shown in Fig. 3. 
Two trees which differ by reversing the order of a node's children or by a sequence of such operations are called dyslectic. Separation coordinates are isometric if and only if the corresponding trees are dyslectic. This gives the recipe how to obtain the quotient (4.3) from $K_{n+1} / \mathbb{Z}_{2}$ : One has to identify those faces labelled by dyslectic trees.

For example, Fig. 4 shows the Stasheff polytope $K_{4}$ with its faces labelled by trees and the corresponding families of separable coordinates. This matches the classical results obtained by Eisenhart [8] and Olevskiu [25]. The reflection symmetry divides $K_{4}$ into two halves, each a quadrilateral. Two adjacent vertices of this quadrilateral are labelled by dyslectic trees and hence have to be identified. One of them is a comb tree, so these are isometric to standard spherical coordinates.
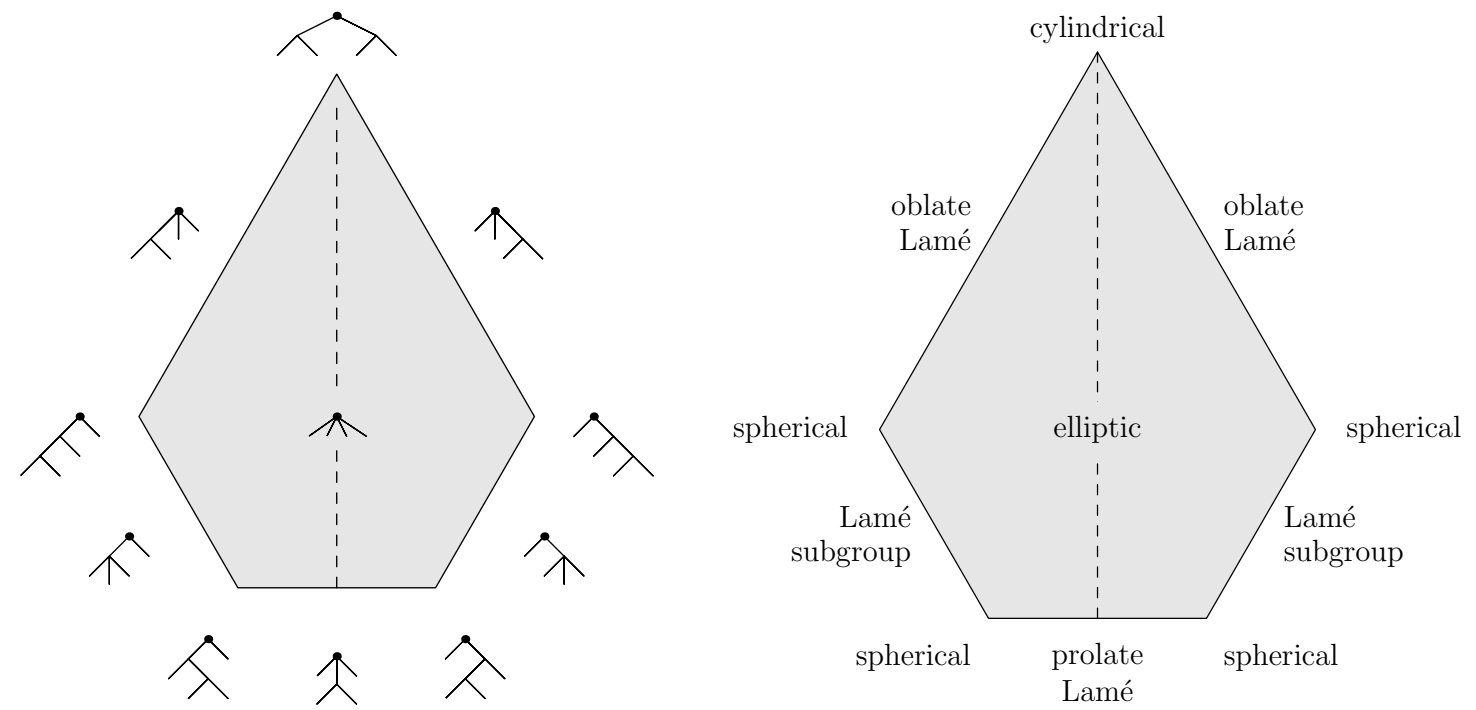

Figure 4. The faces of the Stasheff polytope $K_{4}$, labelled by rooted trees (left) and separable coordinates on $\mathbb{S}^{3}$ (right).

In this way we can not only explain intrinsically the families in [15] and why they can be labelled by trees. The Stasheff polytope also retains the information on how the individual families degenerate into each other. It is, for instance, obvious in our setting that all orthogonal separable coordinates are limits of elliptic coordinates.

\subsection{Operad structure}

A remarkable fact about the moduli spaces $\overline{\mathscr{M}}_{0, n}(\mathbb{R})$, which will allow us to give a simple way how to explicitly construct separable coordinates, is that the sequence $\mathcal{O}(n):=\overline{\mathscr{M}}_{0, n+1}(\mathbb{R})$ carries a natural operad structure, called the mosaic operad [6].

An operad is best explained with composition of functions. Given a function $f$ depending on $k$ arguments and $k$ functions $f_{1}, \ldots, f_{k}$, each depending on an arbitrary (finite) number $n_{i}$ of arguments, we can compose them to give a function $f \circ\left(f_{1}, \ldots, f_{k}\right)$ defined by

$$
\left(f \circ\left(f_{1}, \ldots, f_{k}\right)\right)\left(\boldsymbol{x}_{1}, \ldots, \boldsymbol{x}_{k}\right):=f\left(f_{1}\left(\boldsymbol{x}_{1}\right), \ldots, f_{k}\left(\boldsymbol{x}_{k}\right)\right)
$$

and depending on $n_{1}+\cdots+n_{k}$ arguments. An operad is an algebraic object formalising the properties of this composition: left/right identity, associativity and equivariance.

Definition 5. An operad is a sequence of sets $\mathcal{O}(n)$ indexed by $n=1,2,3, \ldots$ together with a composition map

$$
\begin{aligned}
\circ: \mathcal{O}(k) \times \mathcal{O}\left(n_{1}\right) \times \cdots \times \mathcal{O}\left(n_{k}\right) & \longrightarrow \mathcal{O}\left(n_{1}+\cdots+n_{k}\right), \\
\left(y, x_{1}, \ldots, x_{k}\right) & \mapsto y \circ\left(x_{1}, \ldots, x_{k}\right)
\end{aligned}
$$


and a right action

$$
\begin{aligned}
\star: \mathcal{O}(n) \times S_{n} & \longrightarrow \mathcal{O}(n), \\
(x, \pi) & \mapsto x \star \pi
\end{aligned}
$$

of the permutation group $S_{n}$, satisfying the following properties:

Identity: There is a distinguished element $1 \in \mathcal{O}(1)$ with

$$
y \circ(1, \ldots, 1)=y=1 \circ y .
$$

\section{Associativity:}

$$
z \circ\left(y_{1} \circ x_{1}, \ldots, y_{k} \circ x_{k}\right)=\left(z \circ\left(y_{1}, \ldots, y_{k}\right)\right) \circ\left(x_{1}, \ldots, x_{k}\right) .
$$

\section{Equivariance:}

$$
\begin{aligned}
& (y \star \pi) \circ\left(\left(x_{1}, \ldots, x_{k}\right) \star \pi\right)=\left(y \circ\left(x_{1}, \ldots, x_{k}\right)\right) \star \pi, \\
& y \circ\left(x_{1} \star \pi_{1}, \ldots, x_{k} \star \pi_{k}\right)=\left(y \circ\left(x_{1}, \ldots, x_{k}\right)\right) \star\left(\pi_{1}, \ldots, \pi_{k}\right),
\end{aligned}
$$

where $S_{k}$ acts on $\left(x_{1}, \ldots, x_{k}\right)$ by permutation and $\left(\pi_{1}, \ldots, \pi_{k}\right)$ on $\mathcal{O}\left(n_{1}+\cdots+n_{k}\right)$ under the inclusion $S_{n_{1}} \times \cdots \times S_{n_{k}} \hookrightarrow S_{n_{1}+\cdots+n_{k}}$.

We do not need here the explicit definition of the mosaic operad on the moduli spaces $\overline{\mathscr{M}}_{0, n+1}(\mathbb{R})$. Theorem 3 tells us that there is such a structure on $\mathcal{O}(n):=\mathcal{S}_{0}\left(\mathbb{S}^{n-1}\right)$ as well and once knowing it is there it is not too difficult to figure it out in terms of separable coordinates. Indeed, the sequence of spheres $\mathcal{O}(n)=S^{n-1}$ carries a simple operad structure, given by the composition

$$
\begin{aligned}
S^{k-1} \times S^{n_{1}-1} \times \cdots \times S^{n_{k}-1} & \longrightarrow S^{n_{1}+\cdots+n_{k}-1} \\
\left(\boldsymbol{y}, \boldsymbol{x}_{1}, \ldots, \boldsymbol{x}_{k}\right) & \mapsto \boldsymbol{y} \circ\left(\boldsymbol{x}_{1}, \ldots, \boldsymbol{x}_{k}\right):=\left(y_{1} \boldsymbol{x}_{1}, \ldots, y_{k} \boldsymbol{x}_{k}\right) .
\end{aligned}
$$

By pullback, this defines an operad on (local) coordinate systems. It turns out that this operad restricts to orthogonal separable coordinate systems in normal form, which gives the translation of the mosaic operad from $\overline{\mathscr{M}}_{0, n+1}(\mathbb{R})$ to $\mathcal{S}_{0}\left(\mathbb{S}^{n-1}\right)$. Moreover, the mosaic operad can also be expressed in a simple way in terms of Stäckel systems [30].

The mosaic operad induces an operad on rooted planar trees, whose composition $T \circ\left(T_{1}\right.$, $\left.\ldots, T_{k}\right)$ is given by grafting the $k$ trees $T_{1}, \ldots, T_{k}$ with their respective roots to the $k$ leaves of the tree $T$. Now observe that by iterating this composition any rooted planar tree can be constructed from corolla trees such as the one shown in Fig. 3, which correspond to elliptic coordinates. Consequently, on spheres all orthogonal separation coordinates in a certain dimension can be constructed by composing elliptic coordinates on lower-dimensional spheres under the operad composition (4.4).

Classically, non-generic separable coordinates have been studied via limits of elliptic coordinates, see for example [16]. This requires an intricate analysis, since these limits strongly depend on the path chosen to approach the limit. In our context this is obvious, because the moduli spaces $\overline{\mathscr{M}}_{0, n}(\mathbb{R})$ are iterated blowups. Our result replaces the complicated limiting procedure for elliptic coordinates in a fixed dimension by a simple operad composition of elliptic coordinates from lower dimensions. Likewise, it gives a powerful recursive method for proving statements about separable coordinates and related objects like Stäckel systems: First prove that the statement holds for generic coordinates and then show that it is stable under the operad composition. 


\section{$5 \quad$ Open problems}

From our algebraic geometric point of view on the classification of separable coordinates in conjunction with the example in the preceding section arise a number of natural questions, which we would like to address here.

"The art of doing mathematics consists in finding that special case which contains all the germs of generality."

DAVID HilberT

The proof of Theorem 3 is unsatisfactory in the sense that it does not give an explicit construction of stable algebraic curves with marked points from separable coordinates on spheres, or vice versa. Such a construction would be desirable in view of generalisations of this result (see below).

Problem 1. Give a direct construction of (equivalence classes of) stable algebraic curves with marked points from (equivalence classes of) orthogonal separable coordinates on spheres (or vice versa), realising the isomorphism (4.2).

Finding separable coordinates is only the first step in solving the partial differential equation under consideration. The second is to actually perform the separation of variables. This reduces the partial differential equation in $n$ variables to $n$ ordinary differential equations. The third step is to solve these ordinary differential equations individually (and the fourth to put them together to an explicit solution of the initial equation). The operad structure we found for separable coordinates should therefore be somehow reflected on the corresponding special functions as well.

Problem 2. Find an explicit operad structure on those special functions that arise from a separation of variables on spheres.

Separable coordinates on Euclidean space can be obtained by suitable Inonu-Wigner contractions of separable coordinates on the sphere [12]. In the spirit of our approach, this construction should have an algebraic geometric counterpart, a "contraction of varieties" from $\mathcal{S}\left(\mathbb{S}^{n}\right)$ to $\mathcal{S}\left(\mathbb{E}^{n}\right)$.

Problem 3. Give an algebraic geometric interpretation of Inönü-Wigner contractions of Stäckel systems on spheres.

A posteriori, one can find some clues for the mosaic operad on separable coordinates on spheres in the work of Kalnins and Miller, namely the composition formulas (2.29), (2.45), (2.55) and (3.14) in [15] or (3.45), (3.61) and (3.71) in [13]. For flat and hyperbolic space similar, but more complicated composition formulas are given there as well, cf. formulas (5.17), (4.25) in [15] as well as (5.39), (5.42), (5.47), (5.52), (5.54), (5.60) and (5.62) in [13]. They involve separable coordinates on lower-dimensional constant curvature spaces which are not necessarily of the same curvature. This indicates that there might be an operad structure, or rather a generalisation thereof, for orthogonal separable coordinates on arbitrary constant curvature spaces.

Problem 4. Is there a (generalised) operad on separable coordinates on constant curvature manifolds?

Here we have only considered classical separation of variables, but there exist several flavours of it. In the complex setting one works on a complex instead of a real Riemannian manifold [16]. 
In the conformal setting Killing tensors are replaced by conformal Killing tensors [14]. Our approach adapts to both of these settings, yielding complex and conformal analogues of the variety $\mathcal{S}(M)$. For constant curvature spaces, they all contain $\mathcal{S}\left(\mathbb{S}^{n}\right)$ as a subvariety and therefore extend the moduli spaces $\overline{\mathscr{M}}_{0, n+2}(\mathbb{R})$.

Problem 5. Are the varieties $\mathcal{S}(M)$ for constant curvature manifolds $M$ and their complex or conformal analogues related to moduli spaces of algebro-geometric objects generalising stable algebraic curves of genus zero with marked points?

Another flavour is non-orthogonal separation of variables, for which it is not even clear whether our approach extends to this case as well or not.

Problem 6. Does the set of separable coordinates (orthogonal or not) carry a natural structure of an algebraic variety?

Our Main Theorem holds for any (pseudo-)Riemannian manifold, but all interesting examples we are aware of are for constant curvature manifolds. Trivial examples are manifolds with no or just a single Stäckel system, manifolds of dimension two, for which the Nijenhuis conditions (2.2) are void so that $\mathcal{S}(M) \cong \mathbb{P}^{d-2}$ with $d=\operatorname{dim} \mathcal{K}(M)$, or products $M_{1} \times M_{2}$, for which the inclusion $\mathcal{S}\left(M_{1} \times M_{2}\right) \subseteq \mathcal{S}\left(M_{1}\right) \times \mathcal{S}\left(M_{2}\right)$ is an equality. Possible candidates for non-trivial examples include the Thurston geometries in dimension three and homogeneous spaces, as each of these classes comprises spheres.

Problem 7. Find a non-constant curvature manifold $M$ for which the variety $\mathcal{S}(M)$ has a nontrivial geometry.

The key observation which implied that variable separation is an algebraic geometric problem was that we seek for solutions $K$ of the Nijenhuis integrability conditions within a finitedimensional vector space and that these equations are algebraic in $K$ and its derivatives. This means that our proposed approach is not bound to separable systems, but applies to any problem whose determining equations can be cast into this form. Another example of such a problem which is "covertly algebraic geometric" has recently been found by Jonathan Kress and the author [20], by showing that the set of superintegrable systems in the plane likewise carries the structure of a projective variety equipped with an isometry group action. Here, too, the study of the geometry of this variety reveals hitherto unknown structure in the known classification. We prove, for example, that to every superintegrable system in the plane there is a canonically associated planar arrangement of line triples. So for superintegrable systems hyperplane arrangements seem to play the same role as stable algebraic curves with marked points for separable systems.

Problem 8. Are there further (classification) problems which are "covertly algebraic geometric"? By this we mean that the determining equations are partial differential equations which are polynomial in the variables and their derivatives and for which one seeks all solutions within a finite-dimensional vector space.

\section{Acknowledgements}

This notice is based on a talk held at the workshop "Analytical Mechanics and Differential Geometry" at the Università di Torino on 12th and 13th March 2015 on the occasion of Sergio Benenti's 70th birthday. The author would like to thank the organisers, Claudia Chanu and Giovanni Rastelli, for their kind invitation and hospitality, as well as Willard Miller for valuable discussions on the subject. 


\section{References}

[1] Benenti S., Orthogonal separable dynamical systems, in Differential Geometry and its Applications (Opava, 1992), Math. Publ., Vol. 1, Editors O. Kowalsky, D. Krupka, Silesian University Opava, Opava, 1993, 163184.

[2] Borel A., Linear algebraic groups, Graduate Texts in Mathematics, Vol. 126, Springer, New York, 1991.

[3] Boyer C.P., Kalnins E.G., Winternitz P., Separation of variables for the Hamilton-Jacobi equation on complex projective spaces, SIAM J. Math. Anal. 16 (1985), 93-109.

[4] Davis M., Januszkiewicz T., Scott R., Nonpositive curvature of blow-ups, Selecta Math. (N.S.) 4 (1998), 491-547.

[5] Deligne P., Mumford D., The irreducibility of the space of curves of given genus, Inst. Hautes Études Sci. Publ. Math. 36 (1969), 75-109.

[6] Devadoss S.L., Tessellations of moduli spaces and the mosaic operad, in Homotopy Invariant Algebraic Structures (Baltimore, MD, 1998), Contemp. Math., Vol. 239, Amer. Math. Soc., Providence, RI, 1999, 91-114, math.AG/9807010.

[7] Devadoss S.L., Read R.C., Cellular structures determined by polygons and trees, Ann. Comb. 5 (2001), 71-98, math.CO/0008145.

[8] Eisenhart L.P., Separable systems of Stäckel, Ann. of Math. 35 (1934), 284-305.

[9] Harris J., Algebraic geometry, Graduate Texts in Mathematics, Vol. 133, Springer-Verlag, New York, 1992.

[10] Harris J., Morrison I., Moduli of curves, Graduate Texts in Mathematics, Vol. 187, Springer-Verlag, New York, 1998.

[11] Horwood J.T., McLenaghan R.G., Smirnov R.G., Invariant classification of orthogonally separable Hamiltonian systems in Euclidean space, Comm. Math. Phys. 259 (2005), 670-709, math-ph/0605023.

[12] Izmest'ev A.A., Pogosyan G.S., Sissakian A.N., Winternitz P., Contractions of Lie algebras and separation of variables. The $n$-dimensional sphere, J. Math. Phys. 40 (1999), 1549-1573.

[13] Kalnins E.G., Separation of variables for Riemannian spaces of constant curvature, Pitman Monographs and Surveys in Pure and Applied Mathematics, Vol. 28, Longman Scientific \& Technical, Harlow, John Wiley \& Sons, Inc., New York, 1986.

[14] Kalnins E.G., Miller Jr. W., Conformal Killing tensors and variable separation for Hamilton-Jacobi equations, SIAM J. Math. Anal. 14 (1983), 126-137.

[15] Kalnins E.G., Miller Jr. W., Separation of variables on $n$-dimensional Riemannian manifolds. I. The $n$-sphere $S_{n}$ and Euclidean $n$-space $\mathbf{R}^{n}$, J. Math. Phys. 27 (1986), 1721-1736.

[16] Kalnins E.G., Miller Jr. W., Reid G.J., Separation of variables for complex Riemannian spaces of constant curvature. I. Orthogonal separable coordinates for $\mathrm{S}_{n} \mathbf{C}$ and $\mathrm{E}_{n} \mathbf{C}$, Proc. Roy. Soc. London Ser. A 394 (1984), $183-206$.

[17] Kapranov M.M., The permutoassociahedron, Mac Lane's coherence theorem and asymptotic zones for the KZ equation, J. Pure Appl. Algebra 85 (1993), 119-142.

[18] Knudsen F.F., The projectivity of the moduli space of stable curves. II. The stacks $M_{g, n}$, Math. Scand. 52 (1983), 161-199.

[19] Knudsen F.F., The projectivity of the moduli space of stable curves. III. The line bundles on $M_{g, n}$, and a proof of the projectivity of $\bar{M}_{g, n}$ in characteristic 0, Math. Scand. 52 (1983), 200-212.

[20] Kress J., Schöbel K., An algebraic geometric classification of superintegrable systems on the Euclidean plane, arXiv:1602.07890.

[21] Müller-Hoissen F., Pallo J.M., Stasheff J. (Editors), Associahedra, Tamari lattices and related structures. Tamari memorial Festschrift, Progress in Mathematical Physics, Vol. 299, Birkhäuser/Springer, Basel, 2012.

[22] Mumford D., Geometric invariant theory, Ergebnisse der Mathematik und ihrer Grenzgebiete, Neue Folge, Vol. 34, Springer-Verlag, Berlin - New York, 1965.

[23] Neumann C., De problemate quodam mechanico, quod ad primam integralium ultraellipticorum classem revocatur, J. Reine Angew. Math. 56 (1859), 46-63.

[24] Nijenhuis A., $X_{n-1}$-forming sets of eigenvectors, Indag. Math. 54 (1951), 200-212.

[25] OlevskiüM.N., Triorthogonal systems in spaces of constant curvature in which the equation $\Delta_{2} u+\lambda u=0$ allows a complete separation of variables, Mat. Sb. 27 (1950), 379-426. 
[26] Robertson H.P., Bemerkung über separierbare Systeme in der Wellenmechanik, Math. Ann. 98 (1928), 749-752.

[27] Schöbel K., Algebraic integrability conditions for Killing tensors on constant sectional curvature manifolds, J. Geom. Phys. 62 (2012), 1013-1037, arXiv:1004.2872.

[28] Schöbel K., The variety of integrable Killing tensors on the 3-sphere, SIGMA 10 (2014), 080, 48 pages, arXiv:1205.6227.

[29] Schöbel K., An algebraic geometric approach to separation of variables, Springer Spektrum, Wiesbaden, 2015.

[30] Schöbel K., Veselov A.P., Separation coordinates, moduli spaces and Stasheff polytopes, Comm. Math. Phys. 337 (2015), 1255-1274, arXiv:1307.6132.

[31] Stäckel P., Die Integration der Hamilton-Jacobischen Differentialgleichung mittelst Separation der Variablen, habilitationsschrift, Universität Halle, 1891.

[32] Stasheff J.D., Homotopy associativity of $H$-spaces. I, Trans. Amer. Math. Soc. 108 (1963), 275-292.

[33] Stasheff J.D., Homotopy associativity of H-spaces. II, Trans. Amer. Math. Soc. 108 (1963), $293-312$.

[34] Vilenkin N.Ja., Special functions and group representation theory, Nauka, Moscow, 1965.

[35] Vilenkin N.Ja., Klimyk A.U., Representation of Lie groups and special functions. Vol. 2. Class I representations, special functions, and integral transforms, Mathematics and its Applications (Soviet Series), Vol. 74, Kluwer Academic Publishers Group, Dordrecht, 1993.

[36] Wolf T., Structural equations for Killing tensors of arbitrary rank, Comput. Phys. Comm. 115 (1998), 316-329. 